\title{
Fine structure, magnetic field and heating of sunspot penumbrae
}

\author{
H. C. Spruit ${ }^{1}$ and G. B. Scharmer ${ }^{2}$ \\ 1 Max Planck Institute for Astrophysics, Box 1317, 85741 Garching, Germany \\ e-mail: henk@mpa-garching.mpg.de \\ 2 Institute for Solar Physics, Royal Swedish Academy of Sciences, AlbaNova University Center, 10691 Stockholm, Sweden
}

Received 5 August 2005 / Accepted 12 September 2005

ABSTRACT

We interpret penumbral filaments as due to convection in field-free, radially aligned gaps just below the visible surface of the penumbra, intruding into a nearly potential field above. This solves the classical discrepancy between the large heat flux and the low vertical velocities observed in the penumbra. The presence of the gaps causes strong small-scale fluctuations in inclination, azimuth angle and field strength. The field is nearly horizontal in a region around the cusp-shaped top of the gap, thereby providing an environment for Evershed flows. We identify this region with the recently discovered dark penumbral cores. Its darkness has the same cause as the dark lanes in umbral light-bridges, reproduced in numerical simulations by Nordlund \& Stein (2005, in preparation). We predict that the large vertical and horizontal gradients of the magnetic field inclination and azimuth in the potential field model will produce the net circular polarization seen in observations. The model also explains the significant elevation of bright filaments above their surroundings. It predicts that dark areas in the penumbra are of two different kinds: dark filament cores containing the most inclined (horizontal) fields, and regions between bright filaments, containing the least inclined field lines.

Key words. Sun: sunspots - magnetic fields - convection

\section{Introduction}

The complex magnetic field structure and dynamics in sunspot penumbrae, in fact the very existence of penumbrae, present several outstanding puzzles in solar physics. This fine structure and its dynamics are evidently a consequence of unobservable sub-surface processes that we do not understand theoretically.

One of the foremost theoretical problems associated with sunspot penumbrae (and also umbrae) is the heating problem: The bolometric brightness of the penumbra is some $75 \%$ of the normal solar surface on average; even in the umbra it is still about $20 \%$. Carrying these heat fluxes requires large vertical velocities, of the order $1-2 \mathrm{~km} \mathrm{~s}^{-1}$, which must also be of the right correlation (upward hot, downward cool). The observations do not fit this requirement. The problem is most serious in the umbra, where vertical velocity-intensity correlations are quite low compared to what is needed to carry the observed heat flux (Beckers 1977). The velocities seen in the penumbra are larger, but mostly horizontal (Beckers \& Schröter 1969; Tritschler et al. 2004; Langhans et al. 2005a), with little upward motion in the bright components of the fine structure. There is thus a heat flux problem in the penumbra as well as in the umbra.

One obvious solution to the heat flux problem would be to assume that the penumbra is a very shallow structure, such that the observed heat flux can be carried mostly by radiation from the convection zone below. However, this would imply that the field in the penumbra is nearly horizontal, which is not consistent with the observation that most of the magnetic flux of a sunspot actually crosses the solar surface through the penumbra, not the umbra. The region below the penumbra must be strongly magnetic, as in the quantitative "thick penumbra" models of Jahn \& Schmidt (1994). This rules out the shallow penumbra model of Schmidt et al. (1986). The observations also show that almost all the flux of the penumbra has the same sign as the umbra, and that only a small amount of flux of opposite polarity is in the form of moving magnetic features outside at the penumbra. This rules out the recent model of Thomas et al. (2002), which interprets the structure of the penumbra as due to "turbulent pumping".

In the case of the umbra the heat flow problem has a well known solution: the spot's apparently (at the surface) spacefilling magnetic field actually contains a dense forest of fieldfree gaps below the surface (Parker 1979a). The heat flux of the umbra is channeled through these gaps by field-free convection. The contribution of the present paper is to take the logical step of assuming that the penumbra is equally gappy below its observed surface. We show how, besides solving the heat flow problem, this explains a number of other puzzling observations brought into sharp focus by the recent high-resolution observations with the Swedish 1-m Solar Telescope (Scharmer et al. 2002; Langhans et al. 2005a $\mathrm{a}^{1}$ ).

1 The interpretation of bright filaments as field-free regions just below the surface is in fact old, cf. Mamadazimov (1972). On p134 of this paper, it says: "It seems to us that in all probability the bright interfilamentary elements are parts of the photosphere not covered by the dark filaments of the penumbra". 
A major conceptual advantage of the model is that it provides a much more well defined framework for interpreting the observations than models referring more generically to some kind of magnetoconvection. Before we discuss the model, we briefly review some of the older and more recent observational evidence and the proposed interpretation (Sects. 2, 3, 4).

In Sect. 7 we present a simple potential (current-free) field model for the penumbra, which takes into account field-free intrusions just below the visible surface. We show that this leads to a magnetic field with strong fluctuations in inclination, azimuth angle and strength above the surface without the need to invoke currents in the observed layers. This magnetic field structure is such that it allows locally horizontal or nearly horizontal magnetic fields, thereby providing a natural environment for Evershed flows (for which we do not claim to have an explanation, though we discuss the problem briefly in the discussion section.) We propose that the recently discovered dark cored penumbral filaments (Scharmer et al. 2002) represent the surface manifestation of this sub-surface convection and give observational and theoretical evidence to support this hypothesis.

In the discussion section, we describe some properties of the model that appear consistent with inferred properties of penumbral fine structure and magnetic fields, explain its relations with other models, and make predictions that can be tested with current observational means.

\section{Existing interpretations of penumbral structure}

Despite an overwhelming amount of data accumulated during the last decades, a consistent description of penumbrae, based on observations, has failed to emerge. It appears clear that a major problem in interpreting these data is the small horizontal scales of these structures compared to the spatial resolution of most observations, coupled with apparent rapid gradients with height in both the magnetic field and the Evershed flow. It is now well established that the magnetic field shows strong fluctuations in inclination angle on small scales (e.g. Beckers \& Schröter 1969; Lites et al. 1990; Schmidt et al. 1992; and Title et al. 1993). Furthermore, it is generally agreed that Evershed flows are associated with the more horizontal magnetic fields (e.g. Title et al. 1993) but conflicting evidence exists on the correlation of penumbral intensity fluctuations with the inclination angle of the magnetic field or the Evershed flow. For an extensive discussion of earlier observations, we refer to the the review article of Solanki (2003).

In recent years, polarized spectra as well as measurements of broadband circular polarization, analyzed with inversion techniques, have given incontrovertible evidence for the existence of strong gradients or discontinuities along the line-ofsight in the magnetic field and the Evershed flow, in particular along the vertical direction. This is most clearly demonstrated by the lack of blue-red anti-symmetry of Stokes $V$ profiles, resulting in a net circular polarization when integrated over individual Zeeman sensitive spectral lines, as well as broadband circular polarization when integrated over many such lines.

Sanchez Almeida \& Lites (1992) found that the Stokes profiles of their penumbral spectra required rapid changes in both the inclination of the magnetic field and in the flow speed. Their inversions indicated that the field is progressively more horizontal with depth, coupled with a flow that also increases with depth. These very large variations of the inclination angle with depth, on the order of 45-60', lead Solanki et al. (1993) and Solanki \& Montavon (1993) to infer unacceptably large curvature forces that would be strong enough to destroy the sunspot, if globally present. Solanki \& Montavon (1993) instead proposed a model wherein nearly horizontal flux tubes, imbedded in a more vertical magnetic field, gave rise to the observed net circular polarization. This model avoided the strong curvature (i.e. large changes in the inclination along the magnetic field) that appeared to be indicated by the observations, a problem that was noted already by Sanchez Almeida \& Lites (1992), and emphasized by Solanki et al. (1993).

Sanchez Almeida (1998) pointed to discrepancies in various estimates of inclination changes and claimed that these were consistent only with measurements of broad band circular polarization if the typical scales of inclination changes are on the order $1-15 \mathrm{~km}$. This conclusion was contested by Martinez Pillet (2000) in favor of the imbedded flux tube model, further developed by him and by Schlichenmaier and by Collados (2002), and inspired by simulations of Schlichenmaier et al. (1998a,b). Subsequent investigators, including e.g. Müller et al. (2002), Bellot Rubio et al. (2003, 2004), Borrero et al. (2005), have used forward modelling or inversion techniques to confirm the ability of the embedded flux tube model to explain the observed penumbral Stokes profiles. In the inversions of Borrero et al. (2005) the imbedded flux tube is represented in a very simplistic way, however. The perturbation of the background magnetic field, that is an unavoidable consequence of the imbedded flux tube (Solanki \& Montavon 1993), is ignored. Moreover, only radiation from a single ray going through the center of the flux tube is calculated in the inversions, implying that the flux tube is actually modelled as a flux sheet with constant thicknes and without horizontal boundaries.

It is also clear that inversions applied to observed data do not have a unique interpretation. For example, Westendorp Plaza et al. (2001a,b) found that the magnetic field strength increases with height in the atmosphere, whereas Martinez Pillet (2000) emphasized that observed profiles, obtained at $1^{\prime \prime}$ or lower resolution, are the results of a combination of profiles from very different atmospheric conditions. He demonstrated that the results of the inversions of Westendorp Plaza et al. (2001a,b), referred to above, can be obtained from a flux tube imbedded in a more vertical magnetic field strength having a field strength decreasing with height.

Bellot Rubio et al. (2003, 2004) recorded Stokes spectra in the near infrared obtained from a symmetric sunspot at $40^{\circ}$ heliocentric distance. These authors applied one- and twocomponent inversions to the data and obtained good fits with two separate magnetic field components, one of which was aligned with the Evershed flow, having inclination angles differing by more than $30^{\circ}$ in the outer penumbra.

These investigations provide indisputable evidence for the existence of strong inhomogeneities, in the azimuthal direction as well as along the line-of-sight, in penumbrae. However, they also demonstrate our inability to infer the detailed nature of 
these inhomogeneities from observed Stokes spectra obtained at low spatial resolution. This difficulty is further highlighted by recent work of Borrero et al. (2004), who also inverted their observational data in terms of a one-component model (or rather, a model with components stratified along the line of sight) and a two-component model (in which the data are interpreted as an average of two horizontally separated components). Both kinds of model give very similar results. While this does confirm that a mixture of components is needed, it also makes clear that very little can be deduced about the geometrical ordering of these components.

In their simulations, Schlichenmaier et al. (1998a,b) modelled a penumbral filament as a thin flux tube, based on the siphon mechanism proposed by Meyer \& Schmidt (1968). These simulations show that a flux tube initially located at the magnetopause becomes buoyant as a result of radiative heating by the underlying hotter quiet sun. The tube develops an upflow that bends horizontally and continues outward from the center of the spot. Radiative cooling at the photosphere builds up a pressure gradient that accelerates this flow outwards, explaining the Evershed flow. This is in good overall agreement with inferred observed properties of flux tubes, described above.

Schlichenmaier et al. (1999) estimated cooling times from radiative transfer calculations and used these to interpret bright penumbral grains as the result of hot horizontal flows in flux tubes, cooling off radiatively in the penumbral photosphere. However, these flux tubes can only with the greatest difficulty provide the heat needed to compensate the radiative losses of the entire penumbra. Schlichenmaier \& Solanki (2003) estimated, on the basis of an assumed upflow velocity of $4 \mathrm{~km} \mathrm{~s}^{-1}$, a flux tube diameter of $100 \mathrm{~km}$ and a temperature of the upflow of $12000 \mathrm{~K}$, that such a flow could provide the needed heat along a length of approximately $1200 \mathrm{~km}$, after which the flux tube must exit the penumbra or submerge within it and a new flux tube emerge.

This model thus implies a large amount of magnetic flux dipping down within the penumbra. Whereas there is some evidence for downflows and return flux within the outer penumbra (Westendorp Plaza et al. 1997), this appears to be only a small fraction of the flux emerging through the penumbra (Solanki 2003). The highly resolved magnetograms analyzed by Langhans et al. (2005a) also show very few examples of opposite polarities within penumbrae, of even very large sunspots. There is thus no evidence for significant return flux. Even the opposite polarities observed near the edge of the penumbra account for only a small fraction of the penumbral magnetic flux.

We conclude that although the simulations of Schlichenmaier et al. (1998a,b) provide a plausible scenario for Evershed flows, it appears highly unlikely that such flows can provide the needed uniform heating of the penumbra along its length indicated by observations, and that therefore this is not a likely mechanism for explaining the heating of the penumbra.

In the model proposed by Thomas et al. (2002), field lines emerging in the penumbra are kept submerged outside the spot by turbulent, compressible convection. While such a mechanism might cause local variations in inclination near the edge of the penumbra, it can not explain the large variations in inclination seen throughout the penumbra, up to the transition to the umbra. In particular, they cannot possibly explain the large variations in inclination angle with height over the first one or two hundred $\mathrm{km}$ above the penumbra photosphere required for consistency with observations. Moreover, this mechanism implies a significant amount of flux having opposite polarity to that of the penumbra in the photosphere surrounding sunspots, whereas very small amounts of such flux is actually observed. The turbulent pumping mechanism proposed by Thomas et al. (2002) is not consistent with these observations and can therefore not be responsible for the structure of the penumbral magnetic field.

\section{Recent observations of fine structure of penumbral filaments}

The most striking discovery made so far with the Swedish 1-m Solar Telescope (SST) has been that penumbral filaments consist of a dark core flanked by lateral brightenings (Scharmer et al. 2002). Apart from the potential diagnostics this offers, the discovery indicates that we may be starting to resolve the fundamental scale of penumbra filaments and that important clues to explaining them can be found from highly resolved existing and future images and spectra.

In Fig. 1 we show an example of a sunspot with dark cored filaments. This particular image was recorded with a circular polarizer at the red wing of the $630.2 \mathrm{~nm}$ Fe I line, emphasizing the structure of the filaments. We can see more than 40 dark cored filaments protruding into the umbra but there are also several such filaments that originate in the middle penumbra. Several filaments can be followed over more than $3000 \mathrm{~km}$ and the coherence of these structures along their lengths is striking. Other SST data (Rouppe van der Voort et al. 2003) show examples of filaments that are more than $6000 \mathrm{~km}$ long. In particular, there are no systematic intensity gradients along the length of the filaments shown in Fig. 1. This would be expected if these were individual flux tubes consisting of a hot upflow and a horizontal outflow, proposed by Schlichenmaier \& Solanki (2003) to be responsible for the heating of the penumbra.

Associating the dark cored filaments with individual flux tubes imbedded in a more vertical magnetic field, as suggested by Solanki \& Montavon (1993) is possible, but would imply that such flux tubes must follow very closely the $\tau=1$ surface to be visible over lengths of several $1000 \mathrm{~km}$, and the same must be true for the Evershed flow and the magnetic field within the flux tube. While we do not completely reject the possibility that these structures are individual flux tubes containing siphon flows, we believe that the large radial extension of the dark cored filaments makes this unlikely, we definitely question the suggestion by Schlichenmaier \& Solanki (2003) that such flows provide the heat flux to the penumbra. As discussed by Schlichenmaier \& Solanki (2003) one can also rule out interchange of flux tubes, proposed by Jahn \& Schmidt (1994), as a viable mechanism to heat the penumbra simply on the basis of the long life times of these dark cored filaments, that preserve their identity during more than one hour (Langhans et al. 2005a). 


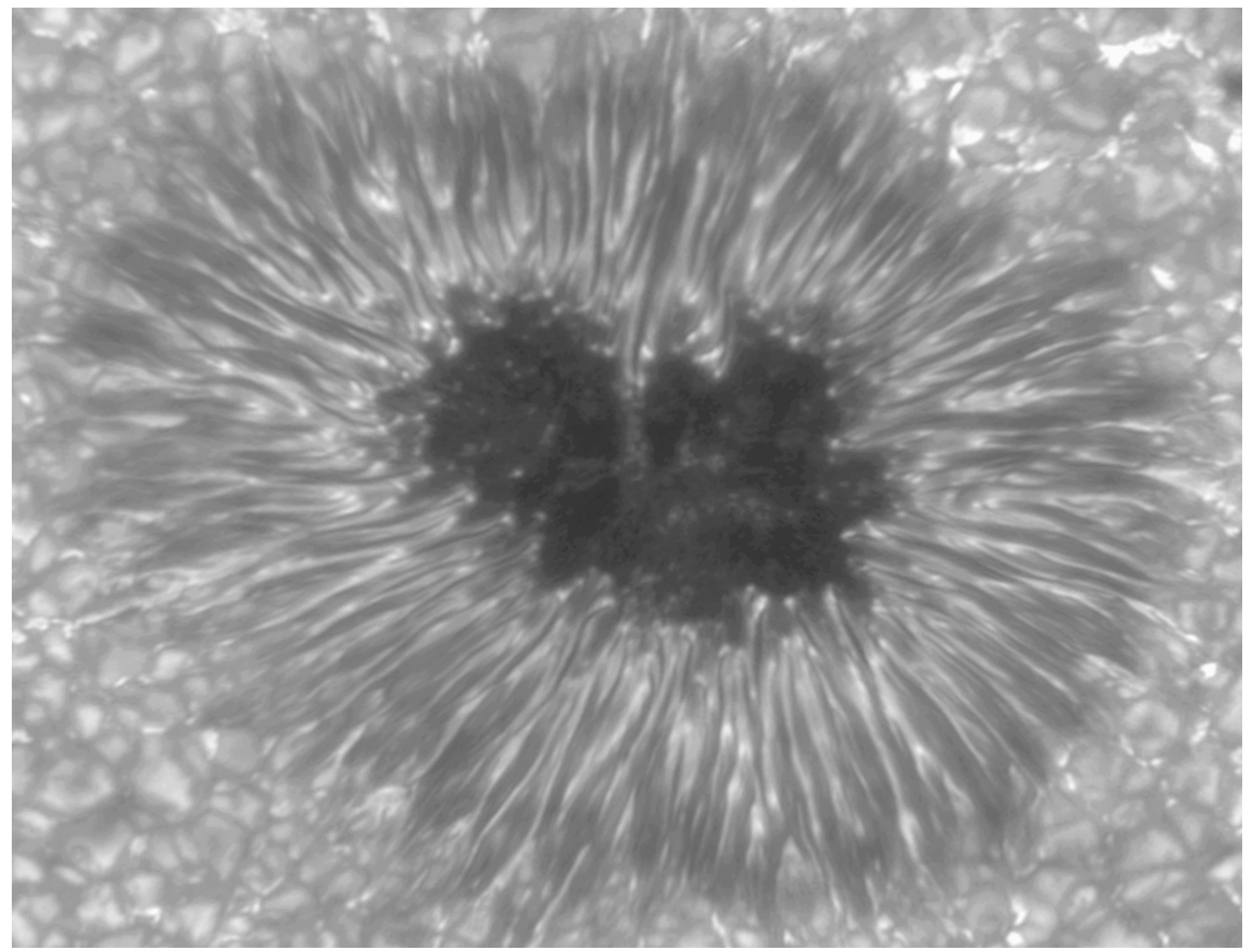

Fig. 1. Sunspot observed with a circular polarizer in the red wing of the $630.25 \mathrm{~nm}$ Fe I line, showing dark cored penumbral filaments with exceptional clarity. More than 40 of these dark cored filaments originate in the umbra, most of them are associated with an umbral dot. (Image recorded by Göran Scharmer and Kai Langhans.)

Figure 2 shows a continuum image of a fairly regular spot recorded at a heliocentric angle of $61^{\circ}$. The disk center (DC) and solar North $(\mathrm{N})$ directions are indicated. This image gives several indications suggesting that the $\tau=1$ surface in the penumbra is not a flat but a corrugated surface. Dark cores are not seen in most of the penumbra. Near the limb side (below "A" marked in Fig. 2), and on the disk center side (below "B"), dark cores are clearly seen in several filaments in the inner part of the penumbra. On the center side, the dark cores are wider and of lower contrast than on the limb side.

At azimuths slightly away from the direction to the limb (below "A", in the inner penumbra), dark cores shift away from the center of bright filaments towards the limb direction. At azimuths around 90 degrees (on the umbra side of "C"), dark cores cannot easily be seen but hints of them are seen as dark streaks separating two nearby filaments. We also note that filaments in a narrow range of azimuth angles around the disk center (above "D") and limb sides (below "A") show markedly sharper structures than the more fuzzy filaments seen away from these directions. This is not an artifact of differential seeing but can be seen consistently in the more than one hour long movie produced from this data set. Finally, we note that filaments near the limb side of the penumbra are associated with dot-like brightenings, as are seen in sunspot images recorded near sun center, but that such brightenings are absent in filaments near the sun center direction. This absence of brightenings on the Sun center side was noted also by Tritschler et al. (2004) in a sunspot only $23^{\circ}$ off disk center.

This and other images of sunspots far away from disk center recorded with the SST show similar evidence of a complex, $\tau=1$ surface where the appearance of the penumbra filaments depends strongly on the azimuth of the viewing angle. Interpreting such images is a delicate problem that is open to personal bias. Here, we give only a partial interpretation by suggesting that we see elevated bright filaments and that the dark cores outline the center and top of these filaments. The suggestion that bright filaments are elevated has been made recently by Schmidt \& Fritz (2004), based on the center-tolimb and azimuthal variation of the penumbra brightness for about 80 sunspots. These authors modeled the penumbra as 


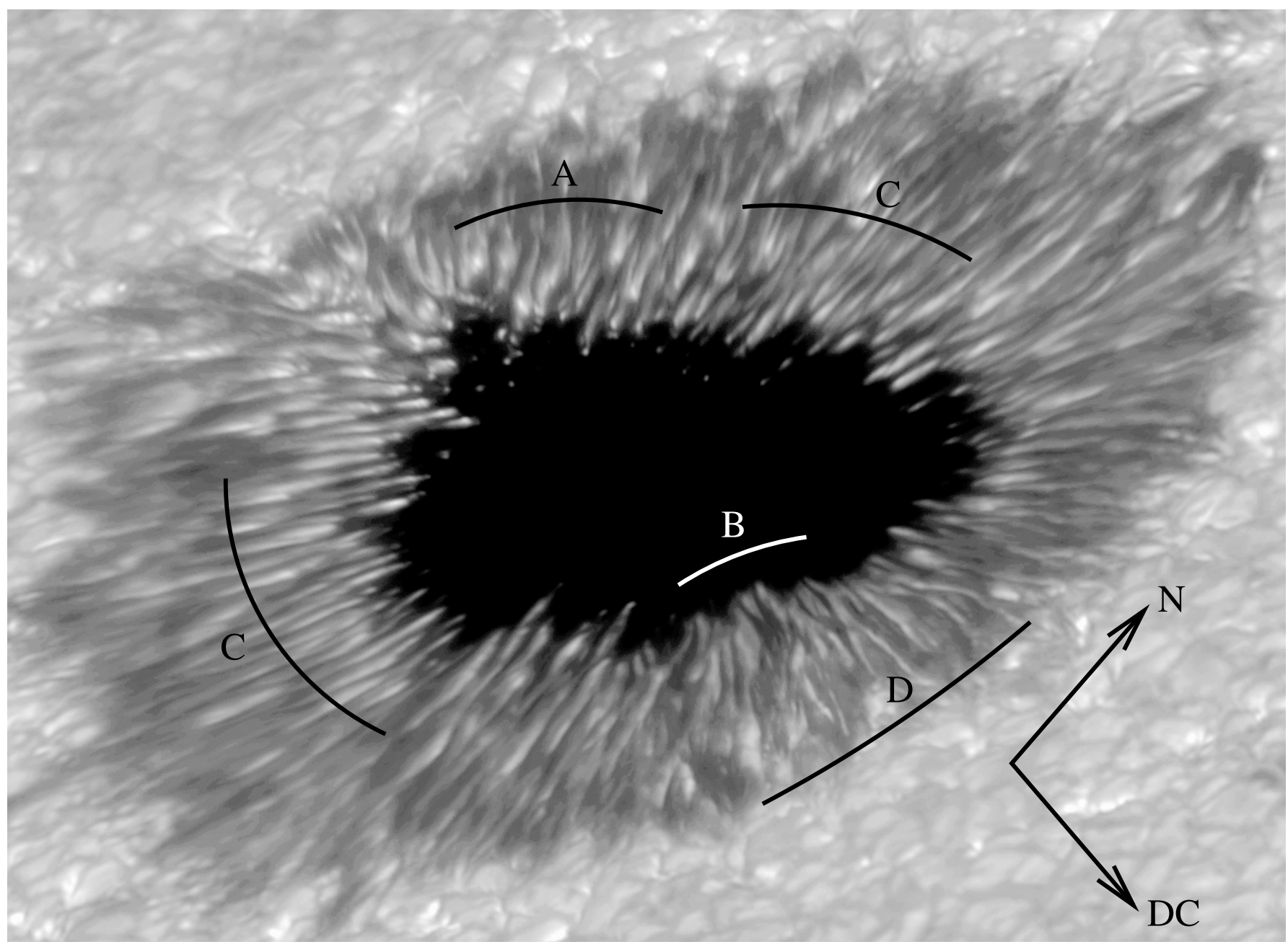

Fig. 2. Evidence for 3D effects in sunspot penumbrae observed at a heliocentric distance of $61^{\circ}$. The disk center (DC) and solar north (N) directions are indicated. (Courtesy of Mats Löfdahl.)

consisting of bright filaments in the shape of elevated bright "boxes" on a dark background. In this simple model that ignores radiative transfer effects, the dark background between the filaments is partly or completely obscured by the bright filaments, leading to modulation of the limb darkening and azimuthal intensity variations. These authors also note that for heliocentric angles larger than $60^{\circ}$, the intensity of the penumbra at the limb side is higher than at the center side. This could be explained as an opacity effect but also by the absence of penumbral brightenings on the disk center side, seen in Fig. 2. The interpretation of dark, but partly optically thin, cores lying "on top" of flux tubes was further supported by Sütterlin et al. (2004) with data from the Dutch Open Telescope (DOT). These authors also noted the prominence of point-like penumbral grains on the limb side, but not center side, of the sunspot observed at a heliocentric distance of only $27^{\circ}$. This, and the results of Tritschler et al. (2004) would indicate that the "head" of a penumbral filament extending into the umbra is inclined by at least some $45^{\circ}$ with respect to the horizontal plane.

Elevation above the background has also been inferred for umbral dots by Lites et al. (1991), who noted the lack of foreshortening of umbral dots observed near the limb. This is consistent with the close connection between umbral dots and penumbral filaments

\section{The connection of penumbral filaments with umbral dots and light bridges}

\subsection{Connection with umbral dots}

The observed continuous evolution of many penumbral filaments into umbral dots (Muller 1973; Scharmer et al. 2002), is illustrated in Fig. 1. This shows more than 40 penumbral filaments protruding into the umbra and in most cases connecting to a peripheral umbral dot. This connection between umbral dots and penumbral filaments suggests common underlying physics.

For the umbra, the most reasonable solution to the heat flux problem is that the heat is supplied in the form of radiation, from a source just below the observed umbral surface. A key indication for this are the umbral dots. In this radiative heating interpretation, a dense pattern of field-free gaps exists just below the umbral surface which communicate with the surrounding 
convection zone. These gaps close just below $\tau=1$, and the heating around their tips is seen as umbral dots. Collectively the dots make up the net heat flux through the umbra.

The existence of these gaps was inferred theoretically (Parker 1979a) from the instability of a vertical magnetic flux bundle to splitting (Meyer et al. 1977), which sets in just below the observed surface. Based on this idea Spruit (1981) has proposed a model for a sunspot in which a bundle of individual thin flux tubes is held together near the base of the convection zone (cf. Spruit \& Roberts 1983). The structure of the spot as seen at the surface in this model reflects the balance between the magnetic buoyancy of the tubes (which keeps them straight and vertical), and their mutual magnetic repulsion at the surface. When the tubes are in temperature equilibrium with their surroundings, their field strength and filling factor can be computed as a function of depth. These results also imply that gaps must exist (filling factor less than 1) just below the umbral photosphere.

The division of a vertical bundle of field lines into slender strands separated by field free convecting plasma is also assisted by the "convective expulsion" process (Zel'dovich 1956; Parker 1963; Weiss 1966). A convecting magnetized plasma tends to separate into field free overturning volumes with the magnetic field concentrated into isolated strands. The strength of these "flux tubes" is just sufficient to resist further tangling and stretching by the convective flow. Once the field has separated into strands, a small amount of fluid motion in their environment is sufficient to keep them concentrated against the action of magnetic diffusion.

The observed connection between umbral dots and penumbral filaments suggest that, just like an umbral dot, a penumbral filament is in fact the surface manifestation of a field-free gap below the observed surface, communicating directly with the surrounding convection zone.

\subsection{The connection with light bridges}

Another discovery made with the Swedish $1 \mathrm{~m}$ telescope was fine structure in umbral light bridges. This fine structure consists both of short, narrow dark lanes oriented roughly perpendicular to the axis of the light bridges and a long narrow dark lane running parallel to this axis (Lites et al. 2004). When viewed near the limb, the fine structure of such light bridges appear asymmetric with respect to the limb and sun center directions, giving the impression of a raised structure against a dark background. Assuming this and by taking advantage of the asymmetry of this structure between the sun center and limb side directions of, Lites et al. (2004) could estimate the apparent height of these structures to be about $300 \mathrm{~km}$. This is of the same order of magnitude as the Wilson depression of sunspots calculated from models (Solanki et al. 1993). Such raised structures in the umbra are expected for structures that have reduced magnetic field strength and thereby increased gas pressure. However, we cannot infer increased gas pressure directly from the fact that a bright structure appears elevated since this may be a consequence simply of the high temperature sensitivity of the $\mathrm{H}^{-}$opacity.
Recently, Nordlund \& Stein (2005) have made 3D radiative magnetohydrodynamic simulations that explain the dark lane running along the main axis of light bridges. These simulations started with two strong magnetic fields of the same polarity on either side of a realistic field-free, convecting photospheric model. After allowing the model to relax from this initial condition and calculating the emerging radiation, a narrow dark lane appears along the symmetry axis between the two magnetic fields. Viewing at different angles of incidence shows this dark lane as a structure located somewhat above the center of the light bridge. The simulations showed it to be unrelated to the convective flow pattern within or near the lane and therefore not a signature of flows. The dark lane is actually a direct consequence of the higher gas pressure in the region between the two magnetic fields which, together with the temperature decrease with height shifts the $\tau=1$ surface upwards to cooler layers. The dark lane forms in the field-free part of the photosphere below the cusp of the magnetic field. It simply outlines a region of enhanced gas pressure compared with the surroundings.

Though light bridges are field-free at the level of the continuum photosphere where the gas pressure can keep the strong fields separate, the rapid decrease of pressure with height implies that these gaps close within some hundred $\mathrm{km}$ above the photosphere, forming a cusp (Fig. 3, middle panel). Since this is only slightly above the surface, magnetic spectral lines formed $100 \mathrm{~km}$ or more above the photosphere should not show indications of a field-free region near the dark lane.

The dark cores of bright penumbral filaments look similar to the dark lanes in light bridges. We take this as our second clue suggesting that they are likewise closely associated with narrow field-free zones between regions of strong magnetic field.

\section{Penumbral energy balance: gaps in an inclined field}

Based mainly on theoretical arguments and primarily the absence of alternative scenarios that explain penumbral heating in accordance with existing data in a satisfactory way, we propose that the penumbra is heated by field-free convection occurring just below the visible surface. We interpret the apparent elevation of penumbral filaments as evidence for enhanced gas pressure, caused by the reduced magnetic field below the centers of such filaments (cf. the sketch in Fig. 3).

Just like the light bridges computed by Nordlund and Stein discussed above, we interpret the dark penumbral cores as signatures of the enhanced gas pressure in the gap compared with the magnetic field surrounding it. The symmetric lateral brightenings seen in penumbrae observed near disk center constitute the bright and inclined sides of the elevated filaments. Their brightness can be interpreted as due to a "bright wall effect" in exactly the same way as in isolated photospheric flux tubes (Spruit 1976, 1977), recently reproduced in realistic 3D radiative magnetohydrodynamic simulations (Carlsson et al. 2004; Keller et al. 2004; Steiner 2005).

The optical depth in the magnetic field bounding the gap is reduced, so that one looks into the field-free walls of the gap, 


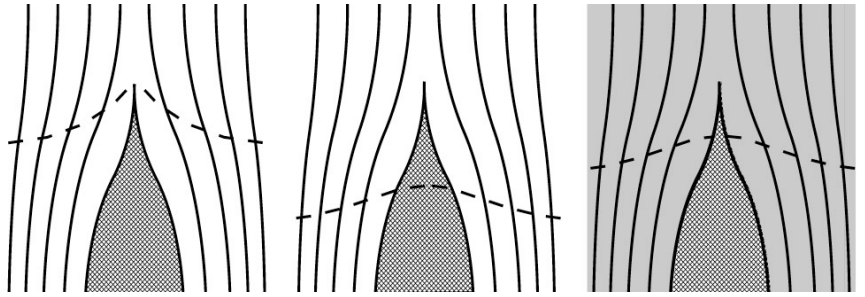

Fig. 3. Gaps (hatched) in a magnetic field near the solar surface (vertical cross-sections). Dashed lines indicate the continuum $\tau=1$ level. The two neighboring flux bundles spread out horizontally above the surface, forming a cusp at some height above $\tau=1$. Left: cusp is located below $\tau=1$, corresponding to an umbral dot. The surface around the gap is brightened by the radiative heat flux. The observed field strength is reduced due to the displacement of field lines by the gap. Middle: a wide gap that would be seen as a field-free "canal" or umbral light bridge. Right: the case of a penumbral filament in the proposed model is like a light bridge, but with an additional horizontal field component (indicated by shading) along the filament.

down to some few hundred $\mathrm{km}$ below the nominal photospheric level. The temperature in the convection zone at that depth is quite high, but as in the case of photospheric flux tubes, radiative cooling of the walls reduces the temperature to something close to normal photospheric temperatures. This explains the observed temperatures of the bright filaments in a way which can be checked quantitatively by numerical simulations like those that have already been done for photospheric flux tubes and light bridges.

The difference between the top and inclined sides of the filaments is dependent on the vertical component of the magnetic field. When this vertical component is weak, as is the case in the outer penumbra, the distinct difference in pressure balance, as well as in optical depth to the gap, between the top and the sides of the filaments is less pronounced. This explains why the dark penumbral cores are seen primarily in the inner and mid penumbra, where the background magnetic field is more vertical.

Through the presence of the magnetic field convection below the surface manifests itself, perhaps somewhat counterintuitively, as a combination of a dark and a bright structure, instead of just a bright structure.

In our model, the main difference between light bridges and penumbral filaments is the presence of a strong horizontal magnetic field component in the penumbra. We note in this context that in the models of embedded flux tubes of Martinez Pillet (2000), the flux tubes were assumed to have a higher pressure and reduced field strength. This gave reduced continuum intensities irrespective of whether the flux tubes were warmer or colder than the surroundings.

Although this interpretation of SST data is subject both to the possibility of personal bias and the strong temperature sensitivity of the $\mathrm{H}^{-}$opacity, we believe that it is a highly plausible scenario which is also strongly supported by the numerical simulations of Nordlund \& Stein (2005). In particular, our model allows uniform heating of filaments along their lengths by convection below the visible surface, thus eliminating the need to provide such heating by horizontal Evershed flows.
The scenario proposed here implies a penumbral magnetic field arranged in the form of radial sheets below the surface, separated by field-free gaps. Above the surface, such a magnetic field must expand and close over the centers of the filaments, so that the field line inclination (angle with respect to the vertical) is increased. For the same reason, the field strength must be somewhat lower than in the surroundings.

A striking property of penumbral filaments is their inward motion, known to represent a pattern motion rather than a fluid displacement (Muller 1973). In terms of our model, this means that the gaps open up progressively towards the umbra. The exact reason for this opening process is a separate, though very interesting, question which we do not attempt to answer in this paper.

\section{Above the surface the field must be nearly current-free}

\subsection{Forces in embedded tube models}

Due to the very rapidly declining pressure in the atmosphere above the $\tau=1$ surface, there are strong limits on the forces that can be present in the magnetic field configuration above the photosphere (recall that the scale height of the atmosphere is only somewhat larger than the spatial resolution of the best images taken with the Swedish 1-m Solar Telescope).

Observations such as those mentioned in section 2 require strong changes in direction and/or strength of the field on small scales. Arbitrary variations in a magnetic field (i.e. restricted only by $\operatorname{div} \boldsymbol{B}=0$ ) on a length scale $L$ are associated with currents of the order $c B /(4 \pi L)$, and volume forces of the order $B^{2} /(8 \pi L)$. The gas pressure in the layers observed in a penumbral magnetogram is too small to balance these forces.

This problem can be alleviated by restricting the kind of variations in the fields. Since the forces are due to currents perpendicular to field lines, they can be minimized by choosing fields that are approximately force free (currents being parallel to the field lines). This restriction, however, conflicts with the most popular models proposed for the inhomogeneities.

In the models of Solanki \& Montavon (1993), Schlichenmaier et al. (1998a), Bellot Rubio et al. (2005) flux tubes are embedded into a background field of a different direction. Such a configuration can not be force free. This is easiest to see if the background field is perpendicular to the tubes, but the argument carries over to general inclination. To accommodate the tube (horizontal, say), the background field (vertical) must be "pushed aside", opening a gap. In order for magnetic forces to be absent, the field strength must be continuous at the boundary between the tube and the background field, and the field strength must also be constant across the tube. At the top and bottom of the tube, this is not possible because the disturbed background field vanishes there. This is more than just a mathematical inconvenience. The imbalance of forces at top and bottom will cause the tube to flatten horizontally and expand vertically. This releases the energy associated with the perturbation induced in the background field. The flattening happens on an Alfvén crossing time: not 
more than a few tens of seconds, for the inferred densities and length scales.

If the tube is allowed to flatten to completion, the process will eventually slow down and a configuration of magnetic sheets of alternating direction is reached. Such a state can be constructed in principle as a force free configuration. Such a sheet-like configuration has been proposed by Martens et al. (1996). This configuration, however, as well as any other forcefree but not current-free configuration, generates a new, equally debilitating problem.

\subsection{Force-free fields}

A force-free configuration has internal torques. In any such field these torques must be taken up by a surface to which the field lines are connected. In an isolated spot, almost all penumbral field lines return to the surface at a large distance from the spot. The number of field lines dipping down into the photosphere at the edge of the penumbra is small compared with the total magnetic flux crossing the surface through the penumbra. At the distance where the penumbral field lines return to the surface, the field strength is too low to take up the torques associated with any significant variations in direction due to field aligned currents in the penumbra.

This argument can be made quantitative as follows. Let $\boldsymbol{B}_{\mathrm{p}}$ be the untwisted field (the field that would be there in the absence of currents), and $\boldsymbol{B}_{\mathrm{t}}$ the component perpendicular to $\boldsymbol{B}_{\mathrm{p}}$ associated with the force-free currents in a flux bundle of width $l$, the torque on the bundle is constant along its length and proportional to $\ell^{3} B_{\mathrm{p}} B_{\mathrm{t}} / 4 \pi$. Since $B_{\mathrm{p}}$ decreases strongly with distance from the center of the spot (like $1 / r^{2}$, so that $\ell \sim r$ ), the constancy of the torque implies that the ratio $B_{\mathrm{t}} / B_{\mathrm{p}}$ increases (as $r$ ) with distance from the spot. This is a manifestation of the well-known fact (e.g. Parker 1979b) that the twist in a forcefree field accumulates at the lowest field strengths encountered along a field line. Thus if something in the penumbra twists field lines around each other, creating force-free currents, the twist thus induced propagates away (at the Alfvén speed) to the place where the field is weak, i.e. into the corona. This makes it hard to maintain much twist in the penumbra itself.

The differences in inclination seen at the edge of the penumbra are already of the order of one radian. If these differences were due to force-free currents, they would have to be even larger outside the penumbra. At a distance of only 2 spot radii, the differences in inclination would already have to be of the order 2 radians. There is little room in the observations for such large variations in inclination. The structure of the superpenumbra as seen in $\mathrm{H}_{\alpha}$ for example does not show evidence of much variation in inclination at all, let alone differences of the order $90^{\circ}$. This problem also becomes apparent immediately as soon as one attempts to extend a force-free field solution to any significant distance from the spot.

The conclusion is that field-aligned currents can not be used to explain the observed variations of field inclinations. These variations must thus be due to currents perpendicular to field lines. Since this is not possible in the low density regions where the spectral lines are formed, the currents must instead be located deeper down. This is in fact the natural solution to the puzzle of mixed field inclinations, as is shown in the following.

\subsection{Varying inclinations in a potential field}

Variations in inclination on small scales as seen in the penumbra are also possible in potential (=untwisted, current-free) fields. By the nature of scalar potentials, irregularities decrease away from their boundaries. If the length scale of the conditions imposed at the boundaries is $L$, the amplitude of the irregularities decreases as $\exp (-z / L)$ with distance $z$ from the boundary. The observed length scale of the irregularities in field inclination in the penumbra is quite small, and their vertical length scale must be equally small if the field is potential. There is, however, very little in the observations that would contradict this. On the contrary, the interpretation of line polarization in spatially unresolved observations discussed above requires strong gradients in inclination over the height of formation of the line as discussed in Sect. 2. This length is of the same order or smaller than the horizontal length scales in the penumbra.

We turn this line of argument around: the observation of strong variations in field inclination then must mean that the observed level in the atmosphere is in fact quite close to the boundary where these irregularities are imposed. This, of course, fits directly with the strong inhomogeneity just below the observed surface which we inferred from the heat flux problem (Sect. 1).

\section{A potential field model}

The above qualitative description can be made more precise with an exact potential field model. Such a field is currentfree, hence has the form $\boldsymbol{B}=\nabla \phi$, where the scalar potential $\phi$ satisfies the Laplace equation $\nabla^{2} \phi=0$. Let $z$ be the vertical coordinate, $y$ the radial horizontal component (perpendicular to the plane in Fig. 3), and $x$ the tangential component (azimuthal in a frame centered on the spot umbra). The model is two-dimensional,

$\partial_{y}=0$

i.e. variations along the length of the penumbral filament are ignored. The boundary conditions defining the field are imposed at infinity and at the surface of the gap. At large distance from the gap the field is uniform. At the surface of the gap the normal component of the field vanishes. We write the field as

$\boldsymbol{B}=\boldsymbol{B}_{\perp}+B_{y} \mathrm{e}_{y}$,

where $\boldsymbol{B}_{\perp}$ is the field in the $(x, z)$-plane, perpendicular to the filament, and $B_{y}$ the parallel component. Consider first the perpendicular components. A simple field satisfying the boundary conditions is

$\boldsymbol{B}_{\perp}=\boldsymbol{B}_{0}+\boldsymbol{B}_{\mathrm{g}}$

where $\boldsymbol{B}_{0}$ is a uniform vertical "background field":

$B_{z 0} \hat{z}$ 
while the field $\boldsymbol{B}_{\mathrm{g}}$, the disturbance due to the presence of the gap,

$B_{\mathrm{g} x}=\partial_{x} \phi ; \quad B_{\mathrm{g} z}=\partial_{z} \phi$,

has the (2D) potential

$\phi=\sin (k x) \exp (-k z)$.

This is a simple periodic potential in the $x$-direction. Note that by virtue of the properties of scalar potentials, the scale of its exponential decrease with height $z$ is tied to the azimuthal separation $L=2 \pi / k$ between the filaments.

Like the background field $\boldsymbol{B}_{0}$, the parallel component is taken to be uniform. At large distance from the gap, the field thus has a constant inclination $\alpha_{0}$ in the $(y, z)$-plane:

$B_{y} /\left.B_{z}\right|_{\infty}=\tan \alpha_{0}$.

The simplicity of this field is made possible by assuming the freedom of specifying a suitable shape for the surface of the gap. Its field lines are shown in Fig. 4.

The model is associated with a discontinuity in the form of a current sheet at the boundary with the field-free region below. While current sheets are a problem when they are used to separate components in a magnetic atmosphere (see Sect. 6.1), in our model the current sheet is an integral part of the physics. It defines the boundary between the field free gaps and the magnetic field, and the forces associated with it serve to satisfy the condition of pressure balance at this interface.

We emphasize that our primary goal is to investigate the consequences of field-free gaps below the surface on the magnetic field above the surface, not to provide a model for the shape of gap itself, which is modeled rather crudely. In particular, it lacks the cusp (cf. Fig. 3) that will be present in a more realistic model.

\subsection{Properties of the model}

The figure shows how the field lines bend around the gap, and how this causes the field lines to be further apart from each other above the gap. This predicts reduced field strengths observed in this region. The figure does not show the component of the field parallel to the filament, which is homogeneous and perpendicular to the plane of the figure.

The top of the gap would be the region we identify with the dark core of the filament. A horizontal field there, close to the continuum level, would also help to explain the Evershed flow. Because of the rapid decline of density with height, this flow must be close to horizontal, covering a vertical extent not exceeding a couple of pressure scale heights. This in turn requires that the optical depth unity surface of an observed filament does not deviate from horizontal by more than about a pressure scale height, at least in its outer penumbra where the Evershed flow is observed. In our model, this is guaranteed because it identifies the surface of the filament with the boundary between the penumbral magnetic field and the convection zone below. Pressure balance across this interface determines its location in depth. The magnetic pressure of the penumbra causes this depth to be depressed below the normal photosphere by not

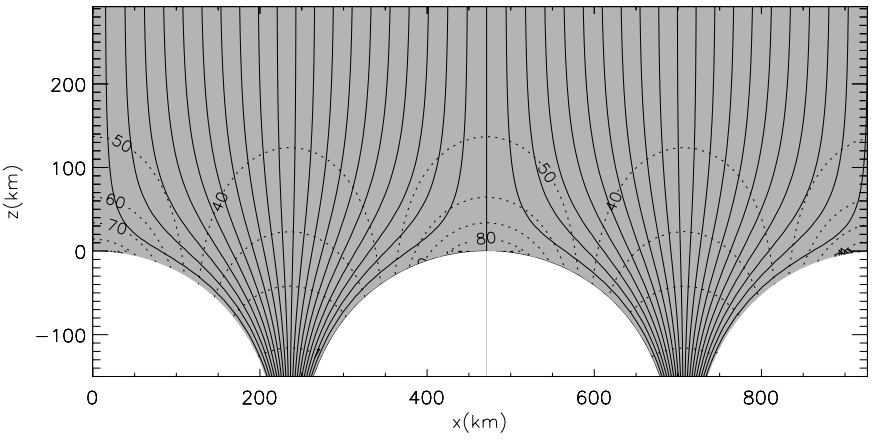

Fig. 4. Idealized field configuration of a penumbral filament. Solid lines show the field lines projected onto a vertical $(x-z)$ plane perpendicular to the filament. The field-free region is shown in white. The $(y$-)component of the field (parallel to the filament, perpendicular to the plane) is indicated by shading. It is uniform outside the gap, such that far from the gap the inclination of the field (angle with respect to the vertical in the $y-z$ plane) is $45^{\circ}$. Dotted lines show contours of constant field line inclination in the $(y, z)$-plane. The higher inclinations above the gap are due to the lower field strength there (cf. Fig. 5). [This model does not contain the cusp region shown in Fig. 3; it will appear, however, in models that take the pressure balance condition into account more realistically, such as those of Nordlund \& Stein (2005).]

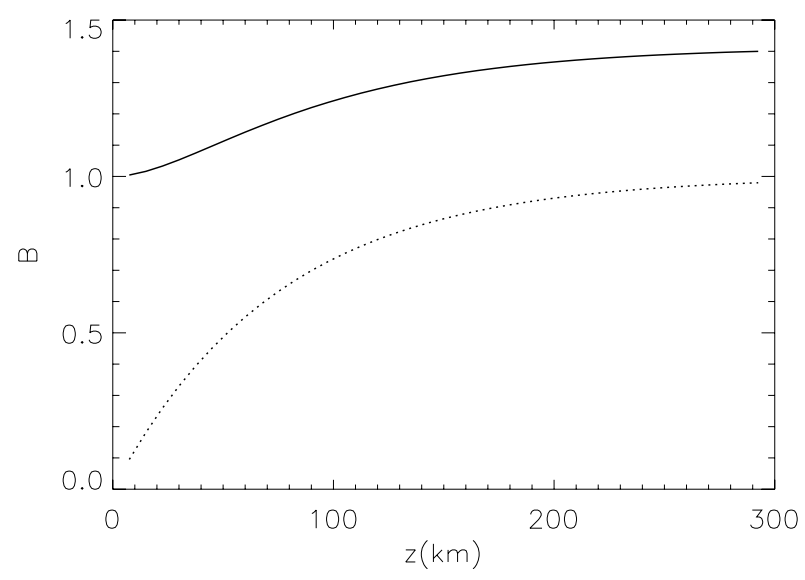

Fig. 5. Variation of the vertical (dotted) and total field strength with height above the middle of the gaps shown in Fig. 4.

more than about one scale height (somewhat more in the inner penumbra where the field strength increases to umbral values). A possible model for the force driving the Evershed flow is the "siphon effect" described by Schmidt \& Meyer.

The field becomes exactly horizontal just at the top of the gap, where the field lines following the sides of the gap meet. To be consistent with observations, this must happen close to the $\tau=1$ layer. This is also consistent with the heat flux requirement mentioned in the Introduction: The observed heat flux can be carried by radiation only if the surface of the gap is close to the continuum $\tau=1$ level (within a few tens of $\mathrm{km}$ ). This in turn implies that the field free gap must contribute to some extent to the formation of spectral lines. This is an intriguing possibility for explaining the "stray light" contributions deduced from observations, as well as the very low field strengths found in the deepest layers in the inversions of Westendorp 
et al., Bellot Rubio et al. (2004), and Borrero et al. (2005) (cf. Sect. 2).

In our analytic model, the shape of the gap is not realistic: its top is approximately circular instead of a cusp as sketched in Fig. 3. This is because the analytic model accounts for the pressure balance between the magnetic field and the gap only in an approximate way. Accurate calculations of field configurations in pressure balance are possible with the methods used for constructing sunspot equilibria (Jahn \& Schmidt 1994), but these are much more demanding.

We finally note that the magnetic field in the penumbra will of course not be exactly a potential field; on the one hand because it is not static, on the other because it must contain material to provide the opacity seen in the lines. This gas exerts a pressure that varies with height, hence introducing some deviations from a potential field. Given the relatively small amounts of gas needed to provide line opacity, these deviations can be small.

Due to the gap, the magnetic field strength above it is reduced. This is evident from the shape of the field lines in Fig. 4, and shown explicitly in Fig. 5. This is also in agreement with the recent results of Langhans et al. (2005a).

\section{Discussion}

We have proposed that bright penumbral filaments are caused by field-free convection just below the surface of the penumbra, and have discussed the theoretical and observational evidence for this interpretation. The field free regions occur as radially oriented gaps in the field that makes up the magnetic flux of the penumbra. We have argued that convection in these gaps between sheets of magnetic field is the most plausible heating mechanism of the penumbra rather than convective flows within a magnetic field.

The existence of such a magnetic field topology is supported by magnetogram data by Langhans et al. (2005a), that consistently show polarization signal in the form of coherent structures extending radially over nearly the entire penumbra.

The field-free gaps below the photosphere intrude into a magnetic fields that is nearly potential (current-free) above the photosphere. This leads to a penumbral magnetic field with large fluctuations on small scales both horizontally and vertically in inclination, azimuth angle, and field strength. In particular, this potential field configuration always leads to nearly horizontal magnetic fields around the tops of the fieldfree gaps. This explains the coexistence of nearly horizontal magnetic fields with highly inclined fields between the bright filaments.

The model is a logical extension of the interpretation of umbral dots as caused by field-free gaps just below the surface (Parker 1979a; Spruit 1981). In this way it also provides a natural explanation for the well-documented evolution of penumbral filaments into umbral dots.

Our model is distinct from the recent proposal by Thomas et al. (2002; see also Weiss et al. 2004; Thomas \& Weiss 2004; Tildesley \& Weiss 2004) in which the penumbral structure is attributed to turbulent pumping by convection. These authors supported their ideas by numerical simulations of an initially horizontal field inside a convectively unstable layer, and propose that a field as seen in these simulations surrounds sunspots. In this proposal the source of the downward pumped magnetic field surrounding the spot are magnetic field lines of the penumbra. Dipping down of these fields lines into the convection zone at the edge of the penumbra is proposed as the cause of the variations in field inclination seen in the penumbra. Such dipping down would be observed in the form of opposite polarity flux surrounding the spot. This does not agree with the observation that very little flux of polarity opposite to that of the spot is ever observed, either inside the penumbra or in the photosphere surrounding sunspots.

Even if an arbitrary amount of downward dipping flux were allowed at the edge, variations in field inclination produced by downward dipping at the edge of the penumbra could not possibly explain the observed inclination variations in the penumbra. Inclination variations as large as $45^{\circ}$ persist all the way to the boundary with the umbra. An even greater difficulty is the very short vertical length scale of these variations, on the order of $200 \mathrm{~km}$. Such a pattern of variations cannot be produced by manipulating the field lines at the edge of the penumbra; they indicate a local origin.

In contrast, the model presented here is built on a mechanism that operates locally and therefore has no problem explaining large inclination changes over a small height range anywhere in the penumbra. In our model, these large fluctuations in inclination angle occur as a natural consequence of field-free gaps below the surface, without the need for any forces acting on the gas above it. This removes the objections of Solanki et al. (1993) concerning the large inclination changes inferred from Stokes spectra by Sanchez Almeida \& Lites (1992). Our model differs from the embedded flux tubes proposed by Solanki \& Montavon (1993) to explain the net circular polarization (NCP) measured in penumbrae. We have shown in Sect. 6.1 that such tubes cannot survive in the observed layers.

Still, the embedded flux model has some similarity to ours by producing strong localized perturbations in the magnetic field, within a few hundred $\mathrm{km}$ above the photosphere. Our model differs mainly in the source of these perturbations, which we identify with field free regions rather than embedded flux tubes. This similarity gives reason to believe that the model presented here will be able to reproduce the observed NCP, as was done successfully with the Solanki-Montavon model.

The model also has some conceptual similarities with that of Schlichenmaier et al. (1998a,b). In both models, heat is carried to the observed surface from the convection zone below by an elongated narrow structure, and some of the dynamics of the flows may also be similar in both. Schlichenmaier's flux tube, however, is endowed with a magnetic field which we argue is actually a hindrance rather than an asset, since it limits the heat flux it can carry.

Models like those proposed in the current literature have a natural pedigree in older thick penumbra models (e.g. Danielson 1961) in which the temperature and magnetic fields are thought of as consisting of a smooth background with fluctuations on it. At a sufficiently rarified level, such "magnetoconvection" type models can be interpreted as being related 
to a maximally inhomogeneous model like our gappy penumbra. We hope to have shown with the present model, however, that such interpretations do not lead very far, and in practice produce obstacles to physical understanding. A major obstacle invited by these interpretations is the intuition of visualizing the low- $\beta$ region above the photosphere in similar terms as the high- $\beta$ regions below, namely as consisting of tubes with identities like those of field line bundles in a high- $\beta$ fluid.

The model proposed here naturally produces a horizontal magnetic field at some height above the gap, thereby allowing for (but not requiring) the existence of horizontal Evershed flows in the visible layers of the photosphere. Such flows are needed in combination with strong gradients or discontinuities in the magnetic field in order to explain the NCP measured in penumbrae. In our model, the heat supplied to these flows is provided by convection below the surface. This also accounts for the fact that the intensity of penumbral filaments is rather uniform along their length.

While still resisting the temptation to claim an explanation of the Evershed flow, we note that our model does put constraints on possible explanations. The field lines wrapping around the gap are sufficiently horizontal to carry a plausible flow only over a finite distance. This implies that the model can only be compatible with flows that are transient and rather local. A possibility that suggests itself is that an Evershed flow may result from a local version of the Schlichenmaier mechanism, operating in the field lines wrapping around the gap. Bundles of these field lines could be heated by interaction with the hot gap. This could produce the same kind of dynamics but on a much more local scale, namely in the boundary layer between the gap and the surrounding field. That the Evershed flow, though smooth and steady on average, is in fact locally transient is suggested by time series of Dopplergrams analyzed by Shine et al. (1994), Rimmele (1994) and time series of spectra by Rouppe van der Voort (2003). These show the Evershed flow as consisting of "velocity packets" repeating irregularly on a time scale on the order of 8-15 min.

Although our model has a horizontal magnetic field near the the top of the gap, this field is quite different from the long horizontal flux tubes simulated by Schlichenmaier et al. (1998a,b). In our model, the origin of the horizontal magnetic field is field lines originating outside the gap and wrapping over the gap towards the center of the filament, where the field becomes aligned in the radial direction. This nearly horizontal field is associated with strong variations in azimuth angle, as well as inclination angle.

In the same way as the dark lanes in light bridges, explained by numerical simulations of Nordlund \& Stein (2005), we identify the dark cores in penumbral filaments as surfaces of enhanced gas pressure, occurring as the result of convection below the visible surface. Recent magnetogram observations by Langhans et al. (2005b), indicate that the magnetic field is more horizontal in the dark cores than in the lateral brightenings. They also report a significantly weaker magnetogram signal in dark cores, which can be interpreted as weaker field strength. From an analysis of spectra recorded in the Fe II $614.9 \mathrm{~nm}$ line, Bellot Rubio et al. (2005) find indications of slightly weaker magnetic fields in the dark cores as compared to the lateral brightenings. This is consistent with our predictions (see Fig. 5).

Recent work by Borrero et al. (2005) confirms the existence of a more horizontal field component with lower than average strength, somewhat higher temperatures, and higher velocities. This is interpreted by these authors in terms of the embedded flux tube model, and advanced as support for it. The strength of this support is limited by the simplicity of the model used to represent the flux tube (a horizontal flux sheet with neglect of perturbation in background field). The observational effects of these components are completely consistent also with our model. This stresses again the ambiguities inherent in multicomponent data inversions.

To resolve these ambiguities more physics needs to be put into the models. In this context the Borrero et al. results give an interesting hint. The low field strength component in their model, envisaged by the authors as horizontally in equilibrium with the other component, must have a higher gas pressure. From their Fig. 8, the difference in gas pressure is about $10^{5} \mathrm{erg} / \mathrm{cm}^{3}$ in the middle of the penumbra. This implies that the low-field component is significantly denser than its environment (the slightly higher temperature has an opposite, but only marginal effect). The two components can therefore not just float at the same level in the atmosphere. The weak-field component will sink unless supported from below by something that is able to exert a pressure of the order of the photospheric value. In our model, this something is of course the field free gap.

We also note that inversions allowing for an unpolarized stray-light component usually come up with an unexpectedly large fraction of unpolarized straylight in the penumbra (e.g. Lites et al. 1993; Westendorp Plaza et al. 2001a). This is to be expected if the line is formed partly in field-free regions. Hence it may well be a signature of the field-free gaps in our model. High stray-light fractions might also be attributed to opposite polarities and consequent cancellation of Stokes profiles (Solanki 2003) if there were a reason for expecting such mixed polarities to be present.

As emphasized by e.g. Martinez Pillet (2000), the ambiguities associated with the inversion and interpretation of low-resolution spectropolarimetric data makes it very difficult to infer penumbral structure from the data alone. The more promising approach is to test sufficiently well-defined physical models such as our present model by forward modeling with detailed radiative transfer calculations.

What is clearly needed are spectropolarimetric data at high spatial resolution. Until such data are available, inversions of low-resolution data incorporating a full "period" of our magnetic field model, with the free parameters it allows, could already constitute a first feasibility test. For future modeling, the strong 3D nature of the penumbra, shown clearly in SST images recorded well away from disk center, should be accounted for. Such modelling will in particular need to explain the strong differences in penumbra fine structure between the limb and the sun center directions.

Acknowledgements. We thank Åke Nordlund, Bruce Lites, Kai Langhans, Mats Löfdahl, Luis Bellot Rubio, Jorge Sanchez and Sami 
Solanki for valuable comments on an earlier version of the text. we thank the anonymous referee for numerous valuable comments. H.S. thanks the Royal Swedish Academy of Sciences for support as visitor of the Telescope. The Swedish 1-m Solar Telescope is operated on the island of La Palma by the Royal Swedish Academy of Sciences in the Spanish Observatorio del Roque de los Muchachos of the Instituto de Astrofısica de Canarias.

\section{References}

Beckers J. M. 1977, ApJ, 213, 900

Beckers, J. M., \& Schröter, E. H. 1969, Sol. Phys., 10, 384

Bellot Rubio, L. R., Balthasar, H., Collados, M., \& Schlichenmaier, R. 2003, A\&A, 403, L47

Bellot Rubio, L. R., Balthasar, H., \& Collados, M. 2004, A\&A, 427, 319

Bellot Rubio, L. R., Langhans, K., Schlichenmaier, R., 2005, A\&A, submitted

Borrero, J. M., Solanki, S. K., Bellot Rubio, L. R., Lagg, A., \& Mathew, S. K. 2004, A\&A, 422, 1093

Borrero, J. M., Lagg, A., Solanki, S. K., \& Collados, M. 2005, A\&A, 436, 333

Carlsson, M., Stein, R. F., Nordlund, Å., \& Scharmer, G. B. 2004, ApJ, 610, L137

Danielson, R. E. 1961, ApJ, 134, 289

Jahn, K., \& Schmidt, H. U. 1994, A\&A, 290, 295

Keller, C. U., Schüssler, M., Vögler, A., \& Zakharov, V. 2004, ApJL, 607, L59

Langhans, K., Scharmer, G. B., Kiselman, D., Löfdahl, M. G., \& Berger, T. E., 2005a, A\&A, 436, 1087

Langhans, K., Scharmer, G. B., Kiselman, D., \& Löfdahl, M. G. 2005b, in prep.

Lites, B. W., Scharmer G. B., \& Skumanich A. 1990, ApJ, 355, 329

Lites, B. W., Bida, T. A., Johannesson, A., \& Scharmer, G. B. 1991, ApJ, 373, 683

Lites, B. W., Scharmer G. B., Berger, T. E., \& Title, A. M. 2004, Sol. Phys., 221, 65

Mamadazimov M., 1972, Sol. Phys., 22, 129

Martens P. C. H., Hurlburt N. E., Title A. M., \& Acton L. W. 1996, ApJ, 463, 372

Martínez Pillet, V. 2000, A\&A, 361, 734

Meyer, F., \& Schmidt H. U. 1968, Z. Ang. Math. Mech., 48, T218

Meyer, F., Schmidt H. U., \& Weiss N. O. 1977, MNRAS, 179, 741

Muller, R., 1973, Sol. Phys., 29, 55

Müller, D. A. N, Schlichenmaier, R., Steiner, \& O., Stix, M. 2002, A\&A, 393, 305

Nordlund, A., \& Stein, R. F. 2005, in prep.

Parker E. N. 1963, ApJ, 138, 552
Parker, E. N., 1979a, ApJ, 234, 333

Parker, E. N. 1979b, Cosmical Magnetic fields (Oxford: Clarendon Press), Chap. 9

Rimmele, T. R. 1994, A\&A, 290, 972

Rouppe van der Voort, L. H. M. 2003, A\&A, 397, 757

Rouppe van der Voort, L. H. M., Löfdahl, M. G., Kiselman, D., \& Scharmer, G. B. 2003, A\&A, 414, 717

Sanchez Almeida, J. 1998, ApJ, 497, 967

Sanchez Almeida, J., \& Lites B. W. 1992, ApJ, 398, 359

Scharmer G. B., Gudiksen B. V., Kiselman D., Löfdahl M. G., \& Rouppe van der Voort L. H. M. 2002, Nature, 420, 151

Schlichenmaier, R., \& Collados, M. 2002, A\&A, 381, 668

Schlichenmaier, R., Jahn K., \& Schmidt H. U. 1998a, ApJ, 493, L121

Schlichenmaier, R., Jahn K., \& Schmidt H. U. 1998b, A\&A, 337, 897

Schlichenmaier, R., Bruls, J. H. M. J., \& Schüssler, M. 1999, A\&A, 349,961

Schlichenmaier, R., \& Solanki, S. K. 2003, A\&A, 411, 257

Schmidt, H. U., Spruit, H. C., \& Weiss, N. O. 1986, A\&A, 158, 351

Schmidt, W., Hofmann, A., Balthasar, H., Tarbell, T. D., \& Frank, Z. A. 1992, A\&A, 264, L27

Schmidt, W., \& Fritz, G. 2004, A\&A, 421, 735

Shine, R. A., Title, A. M., Tarbell, T. D., et al. 1994, ApJ, 430, 413

Solanki, S. K. 2003, A\&AR, 11, 153

Solanki S. K., \& Montavon C. A. P. 1993, A\&A, 275, 283

Solanki, S. K., Walther, U., \& Livingston, W. 1993, A\&A, 277, 639

Spruit, H. C. 1976, Sol. Phys., 50, 269

Spruit, H. C. 1977, Sol. Phys., 55, 3

Spruit, H. C., 1981, in The physics of sunspots; ed. S. Keil, National Solar Observatory, Sunspot, NM 88349, 98

Spruit, H. C., \& Roberts, B. 1983, Nature, 304, 401

Steiner, O. 2005, A\&A, 430, 691

Sütterlin, P., Bellot Rubio, L. R., \& Schlichenmaier, R. 2004, A\&A, 424,1049

Thomas, J. H., Weiss, N. O., 2004, ARA\&A, 42, 517

Thomas, J. H., Weiss, N. O., Tobias, S. M., \& Brummell, N. H. 2002, Nature, 420, 390

Tildesley, M. J., \& Weiss, N. O. 2004, MNRAS, 350, 657

Title, A. M, Frank, Z. A., Shine, R. A., et al. 1993, ApJ, 403, 780

Weiss, N.O. 1966, Proc. Roy. Soc. London A, 293, 310

Weiss, N. O., Thomas, J. H., Brummell, N. H., \& Tobias, S. M. 2004, APJ, 600, 1073

Westendorp Plaza C., del Toro Iniesta, J. C., Ruiz Cobo, B., et al. 1997, Nature, 389, 47

Westendorp Plaza C., del Toro Iniesta J. C., Ruiz Cobo B., et al. 2001a, ApJ, 547, 1130

Westendorp Plaza C., del Toro Iniesta J. C., Ruiz Cobo B., \& Pillet V. M. 2001b, ApJ, 547, 1148

Zel'dovich, Ya. B. 1956, JETP 31, 154 [Sov. Phys. JETP 4, 460 (1957)] 